\title{
Femtosecond x-ray free electron laser pulse duration measurement from spectral correlation function
}

\author{
A. A. Lutman, ${ }^{*}$ Y. Ding, Y. Feng, Z. Huang, M. Messerschmidt, J. Wu, and J. Krzywinski \\ SLAC National Accelerator Laboratory, Menlo Park, California 94025, USA
}

(Received 11 July 2011; published 13 March 2012)

\begin{abstract}
We present a novel method for measuring the duration of femtosecond x-ray pulses from self-amplified spontaneous emission free electron lasers by performing statistical analysis in the spectral domain. Analytical expressions of the spectral correlation function were derived in the linear regime to extract both the pulse duration and the spectrometer resolution. Numerical simulations confirmed that the method can be also used in the nonlinear regime. The method was demonstrated experimentally at the Linac Coherent Light Source by measuring pulse durations down to 13 fs FWHM.
\end{abstract}

DOI: 10.1103/PhysRevSTAB.15.030705

PACS numbers: 41.50.+h, 07.85.Nc, 41.60.Cr

\section{INTRODUCTION}

The Linac Coherent Light Source (LCLS) at SLAC National Accelerator Laboratory is the world's first hard $\mathrm{x}$-ray free-electron laser (FEL) source that began lasing in 2009 [1] and is now in user operation, supporting a wide range of scientific research including physics, structural biology, energy, chemistry, and material science. An FEL $\mathrm{x}$-ray source, such as the LCLS, is based on the so-called self-amplified spontaneous emission (SASE) process. It exhibits three unique properties: ultrafast pulse duration ranging from a few to hundreds of femtoseconds, ultrahigh peak brightness that is many orders of magnitude greater than the brightest storage-ring based synchrotron sources, and nearly full spatial coherence in the transverse directions. Although having these unprecedented characteristics, SASE FEL sources are, however, considered to be chaotic in nature, giving rise to statistical fluctuations in the beam properties on a pulse-by-pulse basis. In particular, the temporal profile of the LCLS FEL x-ray pulse consists of a large number of coherent spikes of varying magnitudes. However, the development of an appropriate diagnostic tool for "seeing" the exact temporal details, including such simple measurement as the pulse duration, has proven to be very challenging and elusive. This is largely due to the vanishingly small cross sections in nonlinear processes at $\mathrm{x}$-ray wavelengths that make temporal correlation techniques, commonly used in the optical regime, exceedingly difficult. The lack of progress in this regard has hindered the LCLS users from knowing the exact pulse duration for resolving atomic/molecular motions and for calculating

\footnotetext{
*aal@slac.stanford.edu
}

Published by the American Physical Society under the terms of the Creative Commons Attribution 3.0 License. Further distribution of this work must maintain attribution to the author(s) and the published article's title, journal citation, and DOI. the exact fluence in studying x-ray nonlinear light-matter interactions.

SASE FEL radiation originates from the initial random distribution of the electrons within a bunch, and its statistical properties are related to the ones of the shot noise. After a start-up phase, FEL enters in the exponential linear regime, where the output power grows exponentially with the undulator length and is proportional to the shot noise power of the electron bunch current. At some undulator length, FEL power does not grow exponentially anymore and reaches saturation. To extract more power from the electrons, postsaturation taper can be applied [2]. After saturation, the relationship between the input shot noise power and the output radiation power is nonlinear.

Statistical fluctuation of the incoherent radiation intensity has been used to get information about the electron bunch length $[3,4]$, as demonstrated by earlier experiments using spontaneous radiation sources [5-8]. However, a SASE FEL differs from a spontaneous source in two aspects. First, although the amplification process in a linear regime does not change the statistical properties of the radiation, it can modify the x-ray pulse duration compared to the electron bunch length due to the exponential gain and slippage effects. Recent studies of the statistical fluctuation and the $\mathrm{x}$-ray pulse duration in the exponential gain regime can be found in Refs. $[9,10]$. Second, a SASE FEL typically operates in the saturation regime for the purposes of intensity stability and a higher power extraction. Here the FEL process is very nonlinear, and it is not obvious whether statistical fluctuations may be useful to retrieve the radiation pulse duration.

In this paper, we present a novel method for measuring the $\mathrm{x}$-ray pulse duration through the analysis of the statistical properties of the SASE FEL spectra. First, we show theoretically how this method can be applied in the exponential growth regime. Then, through numerical simulations, we show why this method still applies in the FEL saturation regime. Finally, we apply this technique to experimental data taken at the LCLS. The measurements 
were performed at various machine settings where the $\mathrm{x}$-ray pulse durations were determined in the range from 10 to $200 \mathrm{fs}$.

\section{THEORY}

The SASE FEL behaves as a narrow band amplifier, which selectively amplifies a wideband random input signal. The fluctuations result from the shot noise of the electron beam current

$$
I(t)=(-e) \sum_{k=1}^{N} \delta\left(t-t_{k}\right)
$$

at the undulator entrance, where the arrival times $t_{k}$ are random variables with the probability density $f(t)$. In our theoretical approach we will use a one-dimensional model assuming that a single transverse mode is established through gain guiding [2]. The spectrum of the FEL electric field $E(t)$ is calculated as $\tilde{E}(\omega)=\int_{-\infty}^{+\infty} E(t) e^{i \omega t} d t$. The first and second order spectral correlation functions are introduced as [11]

$$
\begin{gathered}
g_{1}(\omega, \delta \omega)=\frac{\left\langle\tilde{E}(\omega-\delta \omega / 2) \tilde{E}^{*}(\omega+\delta \omega / 2)\right\rangle}{\sqrt{\left\langle|\tilde{E}(\omega-\delta \omega / 2)|^{2}\right\rangle\left\langle\left.\tilde{E}(\omega+\delta \omega / 2)\right|^{2}\right\rangle}}, \\
g_{2}(\omega, \delta \omega)=\frac{\left\langle|\tilde{E}(\omega-\delta \omega / 2)|^{2}|\tilde{E}(\omega+\delta \omega / 2)|^{2}\right\rangle}{\left\langle|\tilde{E}(\omega-\delta \omega / 2)|^{2}\right\rangle\left\langle\left.\tilde{E}(\omega+\delta \omega / 2)\right|^{2}\right\rangle} .
\end{gathered}
$$

We express a single-shot spectrum taken by the spectrometer as

$$
S(\omega)=\int_{-\infty}^{+\infty} \frac{e^{-\left[\left(\omega^{\prime}-\omega\right)^{2} / 2 \sigma_{m}^{2}\right]}}{\sqrt{2 \pi} \sigma_{m}}\left|\tilde{E}\left(\omega^{\prime}\right)\right|^{2} d \omega^{\prime},
$$

where we assume that the spectrometer resolution function has a Gaussian shape with $\sigma_{m}$ rms width. We define the weighted spectral second order correlation function as

$$
\begin{aligned}
G_{2}(\delta \omega)= & \int_{-\infty}^{+\infty} W(\omega)\left[\frac{\langle S(\omega-\delta \omega / 2) S(\omega+\delta \omega / 2)\rangle}{\langle S(\omega-\delta \omega / 2)\rangle\langle S(\omega+\delta \omega / 2)\rangle}-1\right] \\
& \times d \omega,
\end{aligned}
$$

where the weight function $W$ is described as

$$
W(\omega)=\frac{\int_{-\infty}^{+\infty}\langle S(\omega+b / 2)\rangle\langle S(\omega-b / 2)\rangle d b}{\int_{-\infty}^{+\infty}\langle S(\omega+b / 2)\rangle\langle S(\omega-b / 2)\rangle d b d \omega} .
$$

We introduce a simple model for the exponential growth regime that allows us to obtain an analytical formula for Eq. (5). The process of amplification, within a onedimensional model, can be described by a Green function $h(t, \tau)$, and the electric field can be calculated as the convolution,

$$
E(t)=\int h(t, \tau) I(\tau) d \tau
$$

For electron beams with constant parameters, the SASE FEL Green function has the form [11,12] $h_{t i}(t-\tau)=A_{0}(z) e^{i k_{0} z} e^{-i \omega_{0}(t-\tau)-\left[\left(t-\tau-z / v_{g}\right)^{2} / 4 \sigma_{h t}^{2}\right][1+(i / \sqrt{3})]}$,

where $A_{0}(z) \propto e^{z / l_{g}}$ contains the exponential growth factor, with $l_{g}$ representing the field gain length. The timedependent growth process, originating from an electron beam with a nonflat current profile, can be described assuming that the gain length is a function of $t$ [13]. The gain mechanism also depends on other quantities, such as transverse emittance, energy spread, and undulator taper. To model this, we introduce a time-dependent gain function $h_{t d}$, which is a slow-varying function on the scale of the FEL radiation coherence length. Thus, we express the time-dependent SASE FEL impulse response function as

$$
h(t, \tau)=h_{t i}(t-\tau) h_{t d}(\tau) .
$$

In Appendix A we show that, within the proposed model,

$$
g_{2}(\omega, \delta \omega)=1+\left|g_{1}(\omega, \delta \omega)\right|^{2}
$$

and we derive the analytical formula (A19) for the weighted spectral correlation function (5). When the average spike width is much narrower than the FEL bandwidth $\sigma_{a}=\frac{1}{\sqrt{3} \sigma_{h t}}$, (A19) simplifies to

$$
G_{2}(\delta \omega)=\int_{-\infty}^{+\infty} \frac{e^{\left[-\left(\xi-\delta \omega \xi_{0}\right)^{2} / 2 \sigma^{2}\right]}|\tilde{X}(\xi)|^{2}}{\sqrt{2 \pi} \sigma|\tilde{X}(0)|^{2}} d \xi,
$$

where $\tilde{X}(\omega)$ is the Fourier transform of the average profile

$$
X(t)=\left\langle|E(t)|^{2}\right\rangle
$$

and

$$
\sigma=\sqrt{2} \frac{\sigma_{a} \sigma_{m}}{\sqrt{\sigma_{a}^{2}+\sigma_{m}^{2}}}, \quad \xi_{0}=\frac{\sigma_{a}^{2}}{\sigma_{a}^{2}+\sigma_{m}^{2}} .
$$

Equation (11) can be particularized for different $X(t)$ shapes. For a Gaussian profile with rms duration $\sigma_{t}$, we have

$$
G_{2}(\delta \omega)=\frac{e^{-\left[\delta \omega^{2} \xi_{0}^{2} \sigma_{t}^{2} /\left(1+2 \sigma^{2} \sigma_{t}^{2}\right)\right]}}{\sqrt{1+2 \sigma^{2} \sigma_{t}^{2}}},
$$

and for a flattop profile with full length duration $T$,

$$
G_{2}(\delta \omega)=2 \int_{0}^{1} e^{-\zeta^{2} \sigma^{2} T^{2} / 2}(1-\zeta) \cos \left(\delta \omega \xi_{0} T \zeta\right) d \zeta .
$$

The measurement procedure of the pulse duration is described below. First, a large set of spectra is recorded. By fitting the average spectrum we obtain $\sigma_{a}^{2}+\sigma_{m}^{2}$. Thus, only the spectrometer resolution $\sigma_{m}$ and the pulse duration are unknown. By fitting the experimental $G_{2}$ function with the analytical model, one can finally derive both the pulse duration and the spectrometer resolution. For typical measurement conditions $\sigma_{a} \gg \sigma_{m}, \sigma \approx \sqrt{2} \sigma_{m}, \xi_{0} \approx 1$, and the measurement is insensitive to errors on $\sigma_{a}$. 
Now we would like to compare Eqs. (14) and (15) under the condition that $\sigma_{a} \gg \sigma_{m}$, one can conveniently rewrite the $G_{2}$ function expressions using the variables $\Omega=\frac{\delta \omega}{\sigma_{m}}$, $T_{g}=\sigma_{t} \sigma_{m}$, and $T_{f}=T \sigma_{m}$. In particular, with this notation,

$$
\frac{1}{G_{2}(0)}=\sqrt{1+4 T_{g}^{2}}
$$

gives a measure of the average number of $\mathrm{x}$-ray spectral spikes (coherent modes) within the spectrometer resolution. The number of modes can be approximated by $2 T_{g}$, when $T_{g}$ is large. Mathematical details of the comparison can be found in Appendix B. One can also note that when $T_{f} \gg$ 1 , the method cannot distinguish between Gaussian and flattop pulses having the same rms duration. This is because when $T=\sqrt{12} \sigma_{t}$ the number of modes and the shapes of the $G_{2}$ function are the same [see Fig. 10 and Eqs. (B6) and (B7)]. In this sense the method gives an rms pulse duration measure for compact $\mathrm{x}$-ray pulse shapes, regardless of the shape. For the case $T_{f} \ll 1$, achievable by using a finer resolution spectrometer, the method is able to distinguish between the two shapes. In this case $G_{2}$ is proportional to the square of the modulus of the Fourier transform of the $\mathrm{X}$-ray pulse envelope. Figure 1 represents both described cases for a fixed pulse duration $T_{f}=T_{g} \sqrt{12}$ and for different spectrometer resolutions.

The measurement method described above is based on the exponential growth model introduced in Eqs. (7)-(9). Since, within that model, the FEL behaves as a linear amplifier, the properties of the random Gaussian process of the input signal are retained [14]. Moreover, the expression for the steady-state Green function allows us to derive the analytical relation between the average profile $X(t)$ and the weighted spectral correlation function $G_{2}$.

However, in the saturation regime, Eq. (10) does not hold true in general and we do not have any analytical model such as Eq. (8) to work out an expression for $G_{2}$. The statistical properties in this regime have been studied by numerical simulations in [15], and a simplified analytical model is described in [12]. The most interesting conclusion that can be drawn from these studies is that the quasi-Gaussian statistics is retained for narrow band instantaneous power fluctuations, and that Eq. (10) holds true even at saturation.

Based on the above conclusion, we derived an approximate expression for the $G_{2}$ function without reference to any of the analytical models. The details of the derivation are presented in Appendix C. By assuming that the spectrometer has Gaussian resolution function with rms width $\sigma_{m}$, we obtain

$$
G_{2}(\delta \omega)=\int_{-\infty}^{\infty} \frac{e^{-\Delta^{2} / 4 \sigma_{m}^{2}}|\tilde{X}(\delta \omega+\Delta)|^{2}}{2 \sqrt{\pi} \sigma_{m}|\tilde{X}(0)|^{2}} d \Delta .
$$

The derivation of expression (17) involves several approximations. We assumed that Eq. (10) is valid also in the saturation regime. This result was found in [15] and was also confirmed by our simulations. We considered the case when the average spike width is much narrower than the FEL bandwidth, and the spectrometer resolution width is much narrower than the FEL bandwidth as well. Further, we assume that the approximation $(\mathrm{C} 10)$ is valid. An important result found in [15] is that the spectral correlation functions do not change considerably between the exponential growth and saturation regime. This means that $g_{1}(\omega, \delta \omega)$ has a width in $\delta \omega$ of the order of $1 / T$, where $T$ is the duration of the profile. First, the approximation (C10) requires an assumption that the phase of $g_{1}(\omega+b / 2, \delta \omega) g_{1}^{*}(\omega-b / 2, \delta \omega)$ is nearly zero for $\delta \omega$ being inside the described region, and for $b$ being inside the FEL bandwidth. Second, it requires that the amplitude of $g_{1}(\omega, \delta \omega)$ changes slowly in the $\omega$ variable. This allows
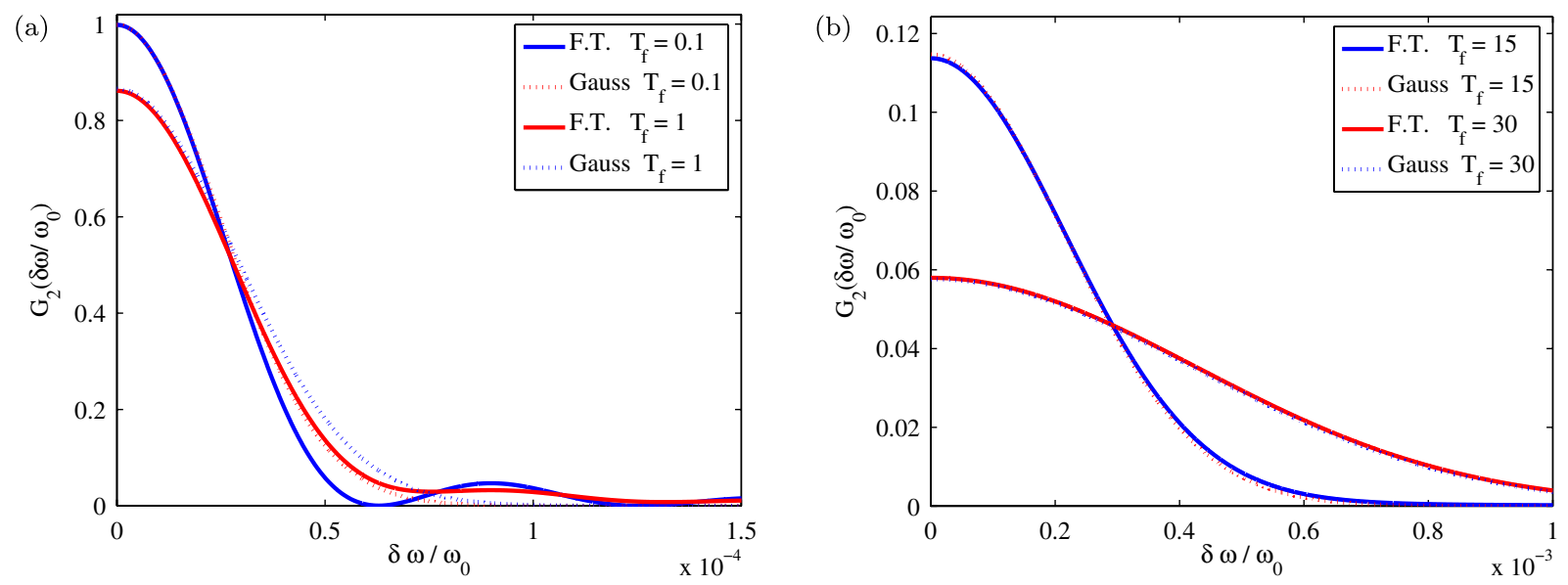

FIG. 1. Comparison between $G_{2}$ functions derived for the flattop model and the Gaussian average x-ray profiles. (a) When $T_{f} \ll 1$, Gaussian and flattop profiles having the same rms duration yield different $G_{2}$ functions. (b) When $T_{f} \gg 1$, Gaussian and flattop profiles having the same rms duration cannot be distinguished. 
us to use the approximation (C10) which is crucial for deriving Eq. (17).

As expected, when $\sigma_{m} \ll \sigma_{a}$ Eq. (11) gives Eq. (17).

\section{NUMERICAL SIMULATIONS}

We ran a series of numerical simulations using a 1D FEL code in order to check if the proposed method is also applicable at saturation and in deep saturation. A flattop electron beam with an energy of $5.9 \mathrm{GeV}$, a peak current of $3 \mathrm{kA}$, and a $1 \mathrm{~mm}$ mrad normalized transverse emittance was used. The undulator has a period of $3 \mathrm{~cm}$ and a strength parameter of 3.5, yielding a radiation wavelength of $0.8 \mathrm{~nm}$ (photon energy $1.55 \mathrm{keV}$ ) and saturation occurring close to

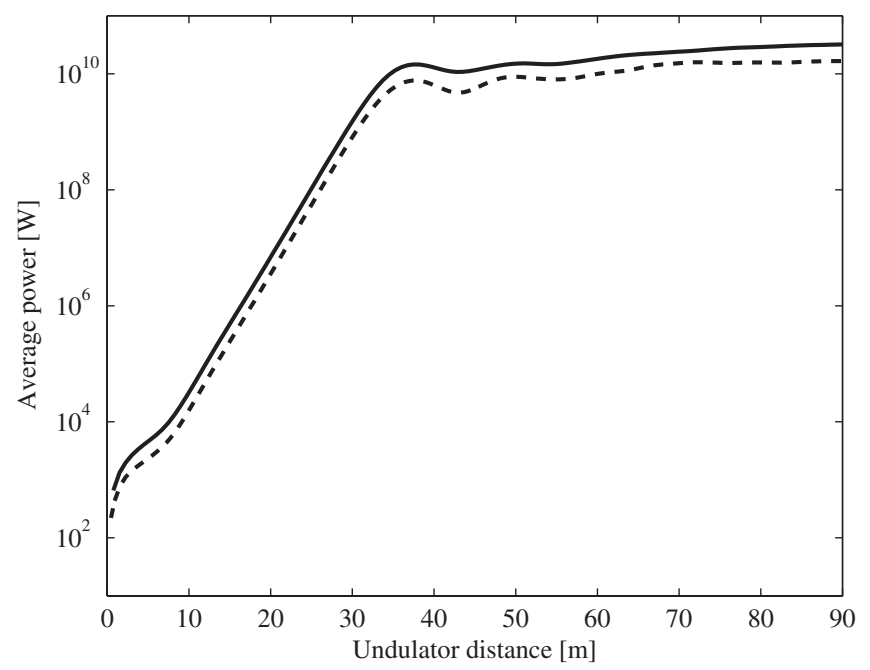

FIG. 2. X-ray pulse average power vs undulator distance. Simulation with the $30 \mu \mathrm{m}$ electron bunch (solid line), simulation with the $3 \mu \mathrm{m}$ electron bunch (dashed line).
$40 \mathrm{~m}$. Two different electron bunch lengths, 30 and $3 \mu \mathrm{m}$, were used during the simulations. Figure 2 shows the $x$-ray pulse power as a function of the undulator distance for each case. Simulation parameters were chosen to be close to the experimental conditions used in Sec. III.

Figure 3 shows that the simulated pulse duration measurements, based on Eq. (17), relate well to the pulse durations obtained directly from the $1 \mathrm{D}$ code. The simulations were performed for both the $30 \mu \mathrm{m}$ and the $3 \mu \mathrm{m}$ electron bunch using 2000 independent shots. In the short bunch case, the slippage and the edge effects played a more important role as compared to the longer bunch, showing that the method works well for ultrashort bunches. Two different relative spectrometer resolutions, $\sigma_{m} / \omega_{0}, 10^{-4}$ and $2 \times 10^{-4}$, were used to show that prior knowledge of the spectrometer resolution is not needed. The relative resolutions have been retrieved as $(1.00 \pm 0.01) \times 10^{-4}$ and $(2.01 \pm 0.05) \times 10^{-4}$ for the long bunch case, and as $(1.00 \pm 0.02) \times 10^{-4}$ and $(1.96 \pm 0.10) \times 10^{-4}$ for the short bunch case.

Simulations starting with a flattop electron bunch show that the method can measure the average pulse duration both in the linear and the nonlinear regime. The pulse duration, however, stays almost constant for different undulator distances. We have also ran simulations with nonflat electron current profiles to prove that the method can actually measure the pulse duration also when the average profile duration changes during the FEL process. We used Gaussian electron bunches with the following parameters: an energy of $5.9 \mathrm{GeV}$, a peak current of $3 \mathrm{kA}$, and a $1 \mathrm{~mm}$ mrad normalized transverse emittance. 500 independent shots were simulated for each electron bunch starting condition. Two rms electron bunch lengths were used: 18 and $6 \mu \mathrm{m}$. The relative spectrometer resolution of $8 \times 10^{-5}$ was used in the simulated measurements.
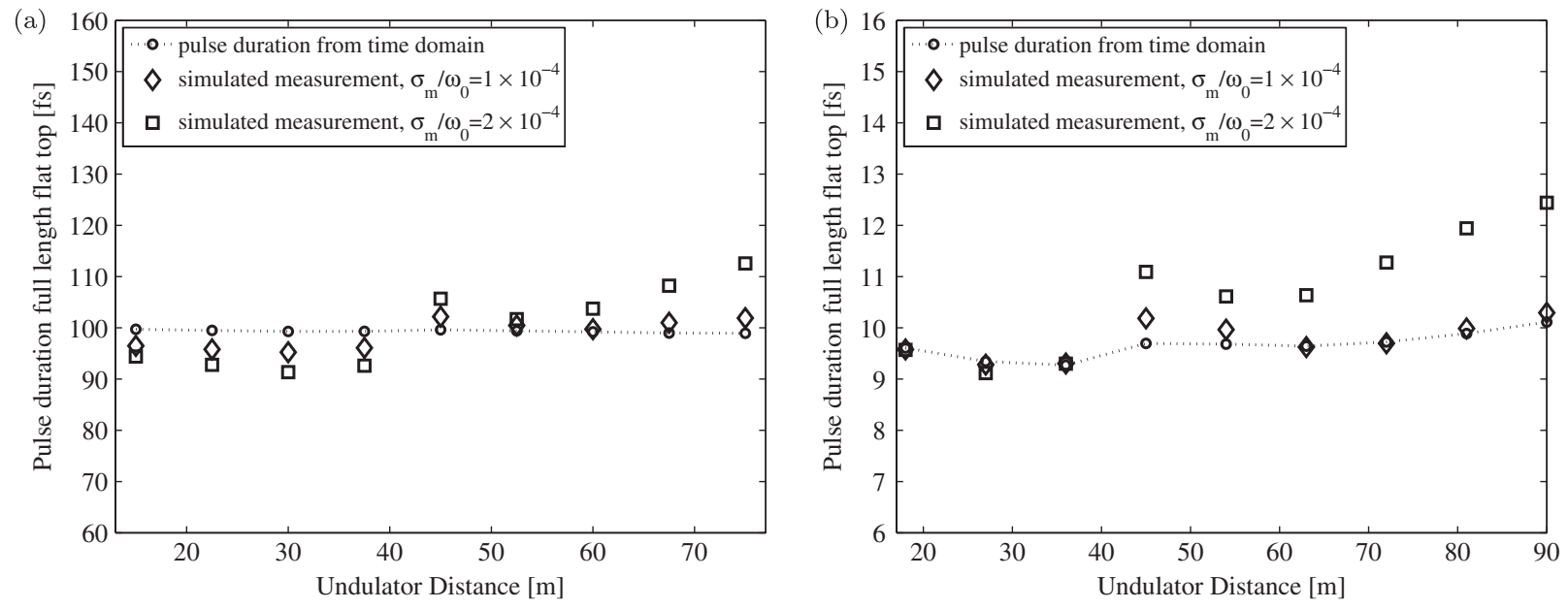

FIG. 3. Simulated measurement of x-ray pulse duration vs undulator distance. (diamonds) Relative spectrometer resolution $\sigma_{m} / \omega_{0}=10^{-4}$, (squares) relative spectrometer resolution $\sigma_{m} / \omega_{0}=2 \times 10^{-4}$. (a) Electron bunch is flattop, $30 \mu \mathrm{m}$ long. (b) Electron bunch is flattop, $3 \mu \mathrm{m}$ long. 

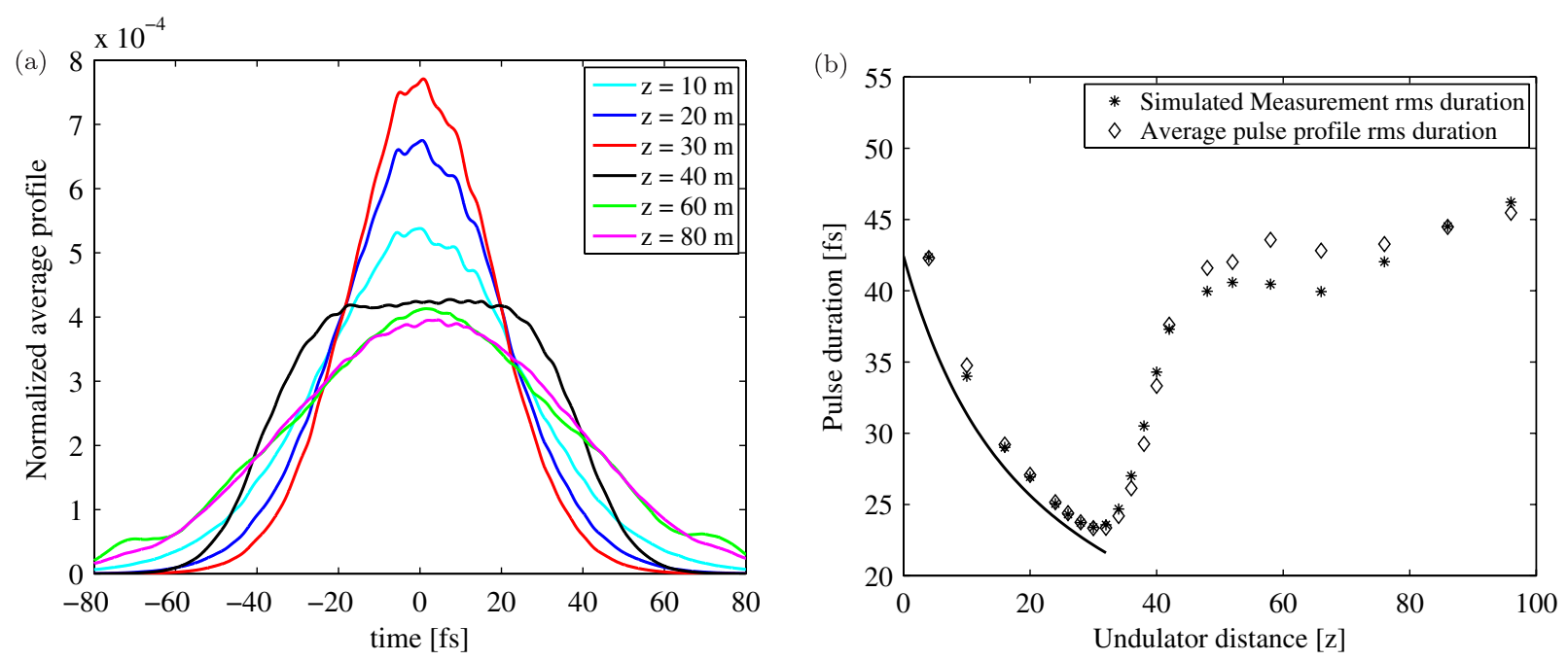

FIG. 4. Results for the Gaussian electron bunch profile with $18 \mu \mathrm{m}$ rms length. (a) Normalized average x-ray profile. A low pass filter has been used to remove the residual spiky structure from the 500 shots average. (b) Pulse duration vs undulator distance. Calculated average profile rms duration (diamonds), simulated measurement rms duration (stars).

The results of the simulated measurements were compared with the rms pulse duration derived directly from the 1D code. Figure 4 shows the comparison for the long bunch case. Figure 4(a) represents the normalized average pulse profiles for different undulator distances. During the exponential growth regime, the selective growth mechanism described by means of the $h_{t d}$ function favors the growth where the density current is higher. This effectively results in a shortening of the x-ray average profile. Figure 4(b) shows the relation between the retrieved pulse duration and the calculated average profile rms duration. We find that the results are in very good agreement both in the exponential growth and in the nonlinear regime.
The solid line in Fig. 4(b) is a quick estimate of the pulse duration obtained by considering the field gain length $l_{g}$ as a function of the current density and neglecting the slippage effects. We can write this formula in a closed form when approximating the gain up to the second order in time:

$$
\sigma_{t}(z)=\sigma_{t}(0) \sqrt{\frac{3 l_{g 0}}{2\left(3 l_{g 0}+z\right)}},
$$

where $l_{g 0}$ is the field gain length for the maximum bunch current. Equation (18) predicts a shortening of the pulse duration during the exponential growth. The retrieved
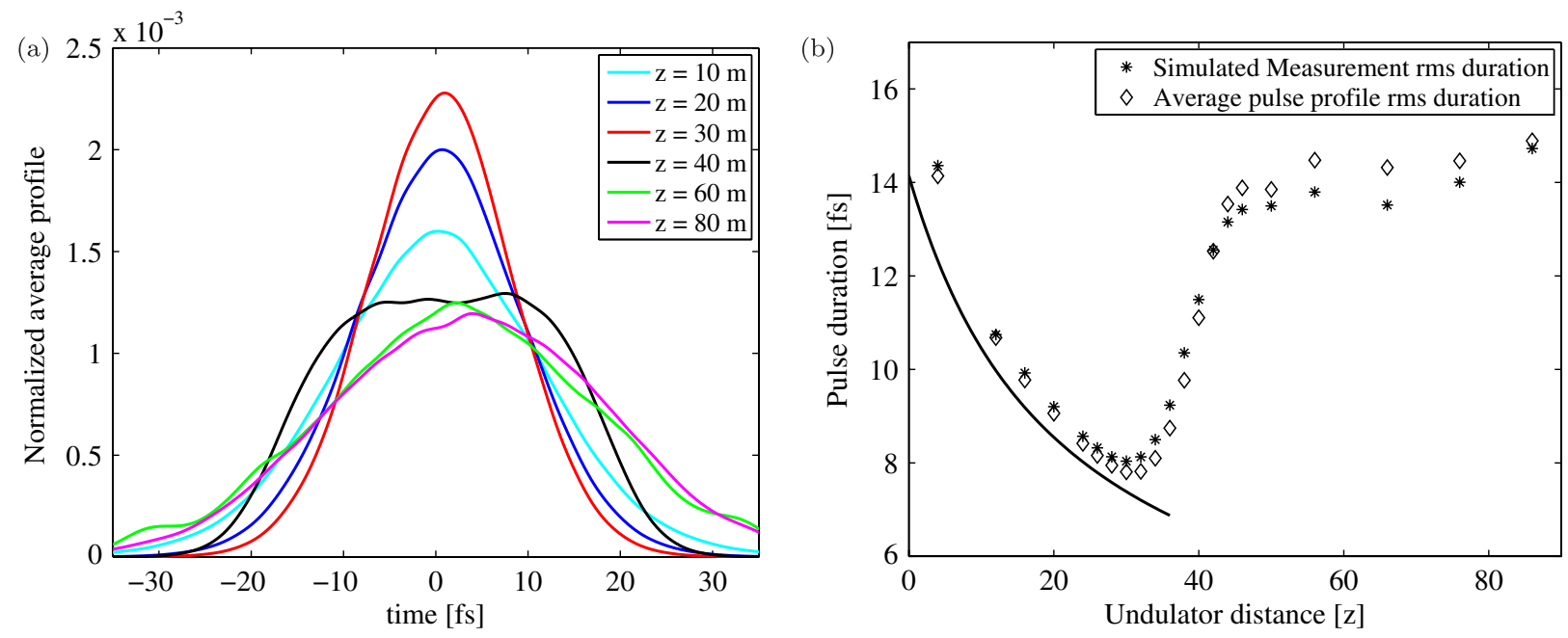

FIG. 5. Results for the Gaussian electron bunch profile with $6 \mu \mathrm{m}$ rms length. (a) Normalized average x-ray profile. A low pass filter has been used to remove the residual spiky structure from the 500 shots average. (b) Pulse duration vs undulator distance. Calculated average profile rms duration (diamonds), simulated measurement rms duration (stars). 
(a)

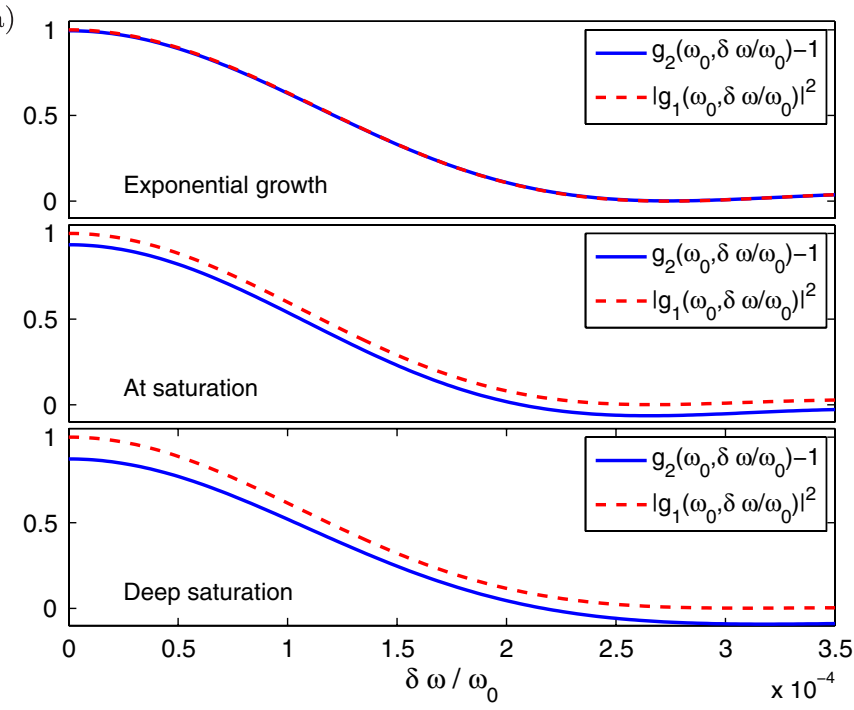

(b)

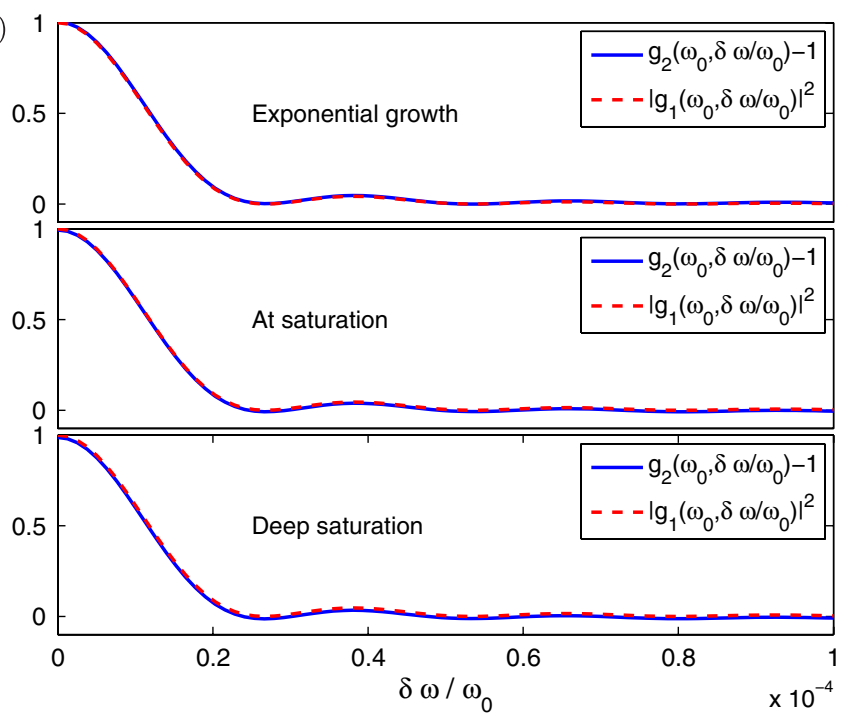

FIG. 6. Equation (10) in the linear regime, at saturation and in deep saturation. $g_{2}\left(\omega_{0}, \delta \omega\right)-1$ (solid blue) and $\left|g_{1}\left(\omega_{0}, \delta \omega\right)\right|^{2}$ (dashed red). (a) Simulated results using an electron bunch $30 \mu \mathrm{m}$ long. (b) Simulation results using an electron bunch $3 \mu \mathrm{m}$ long.

relative spectrometer resolution for this case is $7.90 \times$ $10^{-5} \pm 0.09 \times 10^{-5}$. The simulation starting from the $6 \mu \mathrm{m}$ electron bunch yields shorter pulse durations, which are similar to the shortest measured pulse durations presented in the experimental section. Figure 5(a) shows the normalized average pulse profiles for different undulator distances, and Fig. 5(b) shows the comparison between the retrieved pulse duration and the calculated average profile rms duration. Again we find good agreement between the retrieved pulse duration and the actual $\mathrm{rms}$ duration of the average profile. The retrieved relative spectrometer resolution for the short bunch case is $7.90 \times 10^{-5} \pm 0.11 \times 10^{-5}$.

As expected, our simulations confirm that Eq. (10) holds true in the exponential growth regime. At saturation, the second order correlation function can be empirically approximated as $g_{2}(\omega, \delta \omega) \approx A+B\left|g_{1}(\omega, \delta \omega)\right|^{2}$, when $\delta \omega$ is not too large. This has been confirmed for simulations using different electron bunch current profiles and lengths. Figure 6 shows the comparison between $g_{2}\left(\omega_{0}, \delta \omega\right)-1$ and $\left|g_{1}\left(\omega_{0}, \delta \omega\right)\right|^{2}$ in the nonlinear regime. In saturation, we found $A$ close to 0.88 for the short bunch simulation, and close to 0.99 for the long bunch simulation, while $B$ equals to 1.03 and 1.01 , respectively. This can eventually lead to negative values in Eq. (5), suggesting that it is necessary to fit Eq. (17) with a free offset parameter. This behavior, predicted by the simulations, is confirmed by the experimental data. In the long bunch simulation Eq. (C1) holds better, since the slippage and the edge effects play a less important role. Similar results were found in [15] where the authors explain that, within the accuracy of their simulations, the spectral first and second order correlation functions do not change from the exponential growth regime to the nonlinear regime.

\section{EXPERIMENTAL RESULTS}

The experimental demonstration of the method described above was performed at the LCLS operating at $1.5 \mathrm{keV}$ photon energy. The spectra were recorded by the LCLS soft X-rays spectrometer [16]. For each machine setting around 40000 spectra have been recorded. For each spectrum we have also acquired such quantities as electron beam energy and charge, peak current, trajectory information, and x-ray pulse energy. The latter quantities, particularly the electron beam energy and peak current, were used to select a subset of the recorded data. This subset had aligned spectra and similar electron bunch lengths. A typical subset contained around $5 \%$ of the

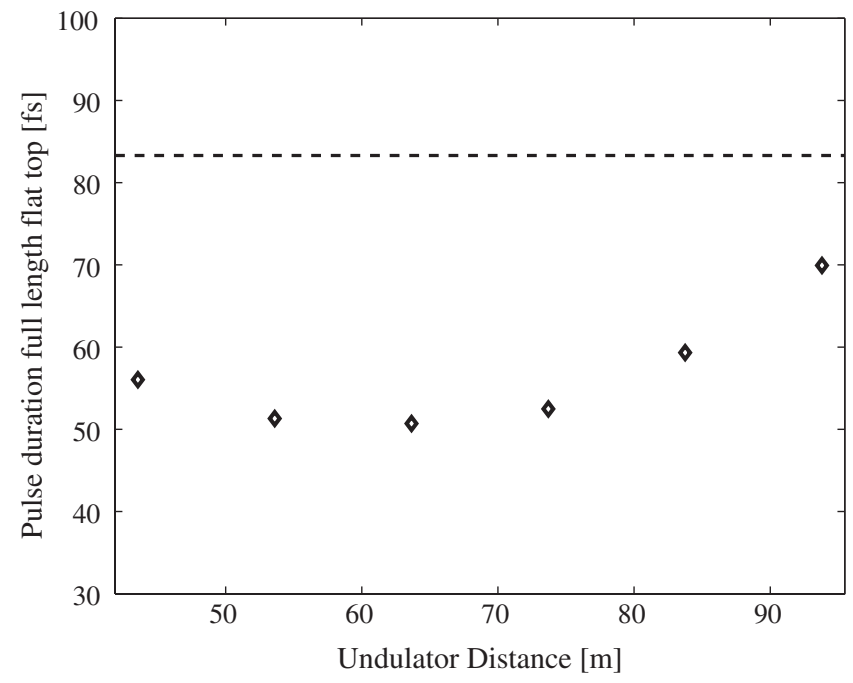

FIG. 7. Measured x-ray pulse duration vs undulator distance (diamonds). Pulse duration expressed as full length flattop. Electron bunch length within the flattop model (dashed). 


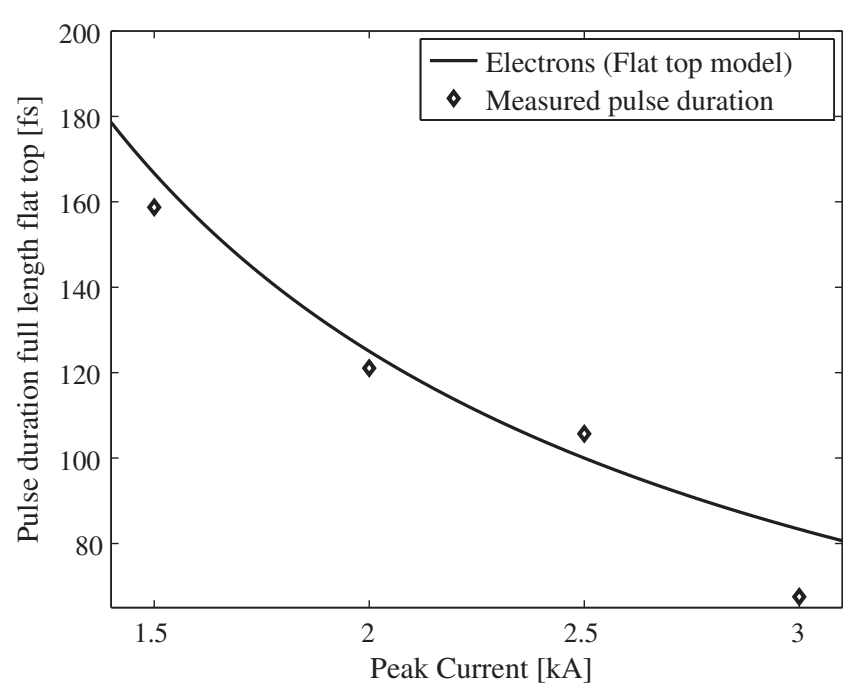

FIG. 8. (Diamonds) Measured x-ray pulse duration vs different peak currents, duration expressed as flattop full length. (Solid) Electrons bunch length for a $250 \mathrm{pC}$ flattop profile as a function of the peak current.

originally recorded results. Since the FEL intensity jitter has an impact on the $G_{2}$ function, measured spectra are normalized by their integral over frequency. Full transverse coherence is assumed for the above analysis. FEL simulations suggest that the transverse coherence decreases after saturation to about 50\%-60\% [17]. Additional analysis of Genesis simulations [18] have shown that the degree of transverse coherence in the vertical and the horizontal directions is $\approx 80 \%$ and $\approx 70 \%$, respectively. This was confirmed by coherence measurements performed during commissioning of the soft x-ray (SXR) instrument [19]. Care must be taken to reduce the effect of the transverse modes on the statistical analysis. The spectra were recorded as 2D images. The spectrometer optics focused the beam only in the vertical direction. During the postprocessing, only the subset of the data on the horizontal coordinate has been used. This is equivalent to using a horizontal slit for improving the transverse coherence in the horizontal direction.

During our experiments we were investigating the influence of different LCLS machine conditions on the statistical properties of measured spectra. We varied the undulator length and the electron bunch peak current. We also applied the slotted foil technique to change the effective electron bunch length that was able to lase [13].

In the first experiment we measured the pulse duration for different undulator lengths. The peak current was set to $3 \mathrm{kA}$, which yields an $83 \mathrm{fs}$ electrons bunch length for a flattop shape. Pulse duration measurements are presented in Fig. 7 showing that $\mathrm{x}$-ray pulses were shorter than electron bunches and that, with our postsaturation taper configuration, the pulse duration increases when the deep saturation is reached. The measured relative spectrometer
TABLE I. Electron bunch length controlled using the slotted foil and measured x-ray pulse duration as FWHM Gaussian. Electron bunch length is calculated with the formula presented in [21]. $\sigma_{m} / \omega_{0}$ is the relative spectrometer resolution measured for each data set.

\begin{tabular}{lcc}
\hline \hline $\begin{array}{l}\text { Estimated unspoiled } \\
\text { electron bunch } \\
\text { length FWHM [fs] }\end{array}$ & $\begin{array}{c}\text { Measured x-ray pulse } \\
\text { duration FWHM [fs] }\end{array}$ & $\begin{array}{c}\text { Measured relative } \\
\text { spectrometer } \\
\text { resolution } \sigma_{m} / \omega_{0}\end{array}$ \\
\hline 10 & 13 & $0.87 \times 10^{-4}$ \\
18 & 24 & $0.92 \times 10^{-4}$ \\
28 & 39 & $0.87 \times 10^{-4}$ \\
56 & 50 & $0.85 \times 10^{-4}$ \\
\hline \hline
\end{tabular}

resolution was similar for the different analyzed data sets, and was equal to $\sigma_{m} / \omega_{0}=(1.02 \pm 0.04) \times 10^{-4}$. The designed spectrometer resolution at $1.5 \mathrm{keV}$ is $0.85 \times$ $10^{-4}$ [16]. Measurements performed at LCLS pointed out that the SXR instrument resolution at that photon energy should be closer to $1.4 \times 10^{-4}$ [20]. The discrepancy could be attributed to the fact that this resolution was derived from an averaged spectra, and it could be influenced by such uncertainties as vibrations and photon beam jitters. The resolution derived by our method is based on the intensity interferometry principle. Therefore, it is much less sensitive to such instabilities. A non-Gaussian shape of the resolution function of the spectrometer could also contribute to the differences in the resolution derived from the two methods.

In the second experiment pulse durations were measured for different peak currents at a fixed electron bunch charge. For these data sets, undulator taper has been applied in order to maximize the output power with 28 undulator segments present. Experimental results have been collected for the peak current from 1.5 to $3 \mathrm{kA}$. Figure 8 shows the measured $\mathrm{x}$-ray pulse duration compared to the electron bunch length in the hypothesis of a flattop electron bunch distribution. Higher peak current electron bunches yield clearly shorter average FEL pulses. Finally, we measured shorter x-ray pulse durations by controlling the electron bunch length using the slotted foil technique [13]. Measured pulse durations obtained by controlling the unspoiled electron bunch length with the slotted foil are found in Table I. The shortest pulse duration was measured for the slotted foil configuration corresponding to an estimated unspoiled electron bunch length of $10 \mathrm{fs}$ FWHM [21]. With this setting, the measured average $\mathrm{x}$-ray pulse duration was 13 fs FWHM. Figure 9(a) shows a typical measured spectrum for different foil settings. It is evident from this figure that the shortest pulses yield spectra with higher fluctuations [Fig. 9(a)] and larger values of $G_{2}(0)$ [Fig. 9(b)]. This figure also shows that the analytical model can fit the experimental data well. 

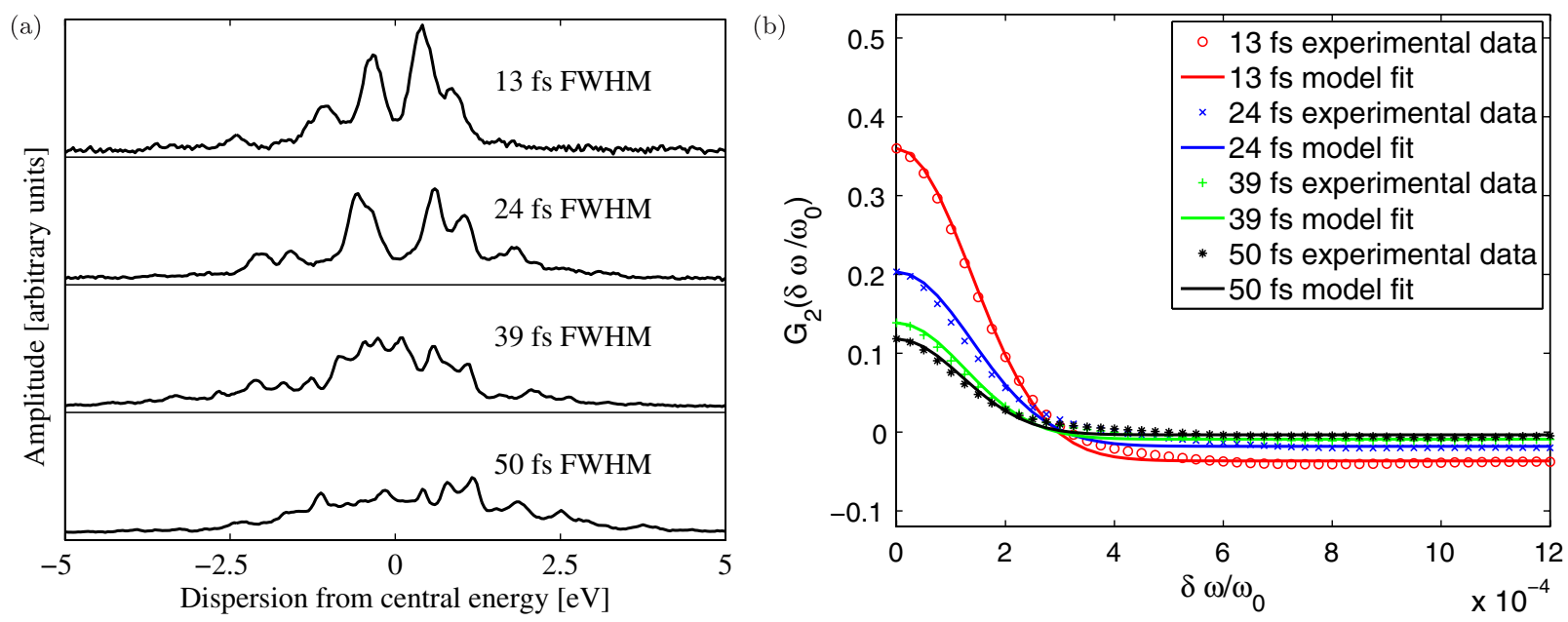

FIG. 9. Pulse duration measurement controlling the bunch length using the slotted foil. (a) Typical measured spectra. Shorter x-ray pulses present spectra with larger amplitude fluctuations. (b) Experimental $G_{2}$ function for different pulse durations (FWHM Gaussian) and fitting with the theoretical model.

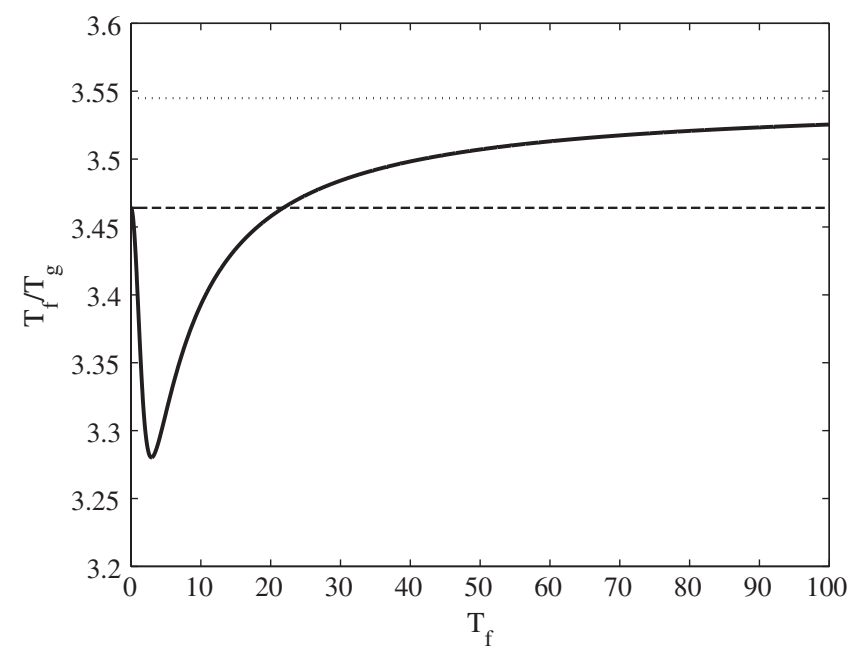

FIG. 10. $\frac{T_{f}}{T}$ as function of $T_{f}$ giving the same value for $G_{2}^{g}(0)=G_{2}^{f^{\prime \prime}}(0)$ (bold solid), $\sqrt{12}$ (dashed), $2 \sqrt{\pi}$ (dotted). $\frac{T_{f}}{T_{g}}=$ $\sqrt{12}$ is a good approximation to have the same value $G_{2}(0)$ for both the flattop and the Gaussian models.

\section{CONCLUSION}

We have developed an effective approach for measuring the average length of ultrafast $\mathrm{x}$-ray pulses from SASEbased FEL sources by using the statistical characteristics not in the time, but in the spectral domain. This technique was shown to be experimentally applicable not only in the exponential growth region, but also in the nonlinear region of the SASE amplification process as confirmed by analyzing simulated data sets. We observed that the extracted $\mathrm{x}$-ray pulse duration varied consistently with the manipulated electron bunch length. The hypothesized evolution of the pulse duration as a function of the undulator distance was also observed for the first time, lending further credence to our analysis. In addition, this method can be used to measure the resolution function of a spectrometer as a cross-check to other direct experimental techniques, such as using an absorption line. We believe that our method can be extended to other SASE-based FELs at arbitrary wavelengths. This approach can be also considered for the analysis of any chaotic process where the output signal originates from a nonlinear, narrow band amplification of a Gaussian process.

\section{ACKNOWLEDGMENTS}

We wish to thank P. Emma, W. Schlotter, J. J. Turner for assistance in the soft x-ray spectrometer experiments, H. Durr, W. Fawley, P. Heimann, and J. Stöhr for useful comments and discussions. This work was supported by Department of Energy Contract No. DE-AC0276 SF00515.

\section{APPENDIX A: $G_{2}$ FUNCTION DERIVATION}

In this Appendix we derive an expression for the weighted second order correlation $G_{2}$ function defined as

$$
G_{2}(\delta \omega)=\int_{-\infty}^{+\infty} W(\omega)\left[\frac{\langle S(\omega-\delta \omega / 2) S(\omega+\delta \omega / 2)\rangle}{\langle S(\omega-\delta \omega / 2)\rangle\langle S(\omega+\delta \omega / 2)\rangle}-1\right] d \omega
$$

with 


$$
W(\omega)=\frac{\int_{-\infty}^{+\infty}\langle S(\omega+b / 2)\rangle\langle S(\omega-b / 2)\rangle d b}{\int_{-\infty}^{+\infty}\langle S(\omega+b / 2)\rangle\langle S(\omega-b / 2)\rangle d b d \omega}
$$

where $S(\omega)$ is a single-shot spectrum collected after a spectrometer. For a spectrometer with a Gaussian resolution function having rms width $\sigma_{m}$, the single-shot spectrum is denoted as

$$
S(\omega)=\int_{-\infty}^{+\infty} \frac{e^{-\left[\left(\omega^{\prime}-\omega\right)^{2} / 2 s_{m}^{2}\right]}}{\sqrt{2 \pi} s_{m}}\left|\tilde{E}\left(\omega^{\prime}\right)\right|^{2} d \omega^{\prime}
$$

and Eq. (A1) can then be written as

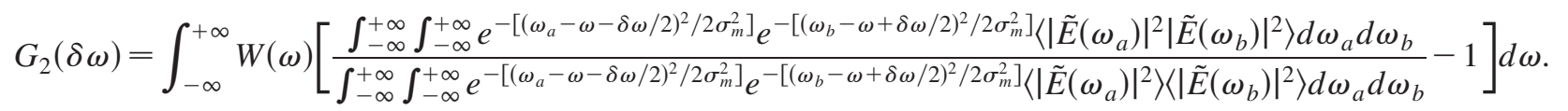

With a substitution, $\left\{\omega_{a}, \omega_{b}, \omega\right\} \rightarrow\{\Omega, \Delta, \omega\}, \omega_{a}=\omega+\Omega+\delta \omega / 2+\Delta / 2, \omega_{b}=\omega+\Omega-\delta \omega / 2-\Delta / 2$, and $\omega=\omega$, we can write Eq. (A4) as

$$
\begin{aligned}
G_{2}(\delta \omega)= & \int_{-\infty}^{+\infty} W(\omega)\left[\frac{\int_{-\infty}^{+\infty} \int_{-\infty}^{+\infty} e^{-\Delta^{2} / 4 \sigma_{m}^{2}} e^{-\Omega^{2} / \sigma_{m}^{2}}\left\langle|\tilde{E}(\omega+\Omega+\delta \omega / 2+\Delta / 2)|^{2}|\tilde{E}(\omega+\Omega-\delta \omega / 2-\Delta / 2)|^{2}\right\rangle d \Delta d \Omega}{\int_{-\infty}^{+\infty} \int_{-\infty}^{+\infty} e^{-\Delta^{2} / 4 \sigma_{m}^{2}} e^{-\Omega^{2} / \sigma_{m}^{2}}\left\langle|\tilde{E}(\omega+\Omega+\delta \omega / 2+\Delta / 2)|^{2}\right\rangle\left\langle|\tilde{E}(\omega+\Omega-\delta \omega / 2-\Delta / 2)|^{2}\right\rangle d \Delta d \Omega}-1\right] \\
& \times d \omega .
\end{aligned}
$$

Let us derive a particular expression for $G_{2}$ for the exponential growth regime by using the model based on Eqs. (8) and (9). The Fourier transform of the electric field is evaluated as

$$
\tilde{E}(\omega)=\int_{-\infty}^{+\infty}(-e) \sum_{k=1}^{N} h_{t i}\left(t-t_{k}\right) h_{t d}\left(t_{k}\right) e^{i \omega t} d t=(-e) \tilde{H}_{t i}(\omega) \sum_{k=1}^{N} e^{i \omega t_{k}} h_{t d}\left(t_{k}\right)
$$

where $\tilde{H}_{t i}(\omega)=\int_{-\infty}^{+\infty} h_{t i}(t) e^{i \omega t}$. First we show the validity of Eq. (10) by following an approach similar to the one described in [11]. We calculate the first order spectral correlation as

$$
\begin{aligned}
\left\langle\tilde{E}(\omega+\delta \omega / 2) \tilde{E}^{*}(\omega-\delta \omega / 2)\right\rangle= & e^{2} \tilde{H}_{t i}(\omega+\delta \omega / 2) \tilde{H}_{t i}^{*}(\omega-\delta \omega / 2)\left\langle\sum_{k=1}^{N} \sum_{j=1}^{N} h_{t d}\left(t_{k}\right) h_{t d}^{*}\left(t_{j}\right) e^{i(\omega+\delta \omega / 2) t_{k}} e^{-i(\omega-\delta \omega / 2) t_{j}}\right\rangle \\
= & e^{2} \tilde{H}_{t i}(\omega+\delta \omega / 2) \tilde{H}_{t i}^{*}(\omega-\delta \omega / 2)\left(\left\langle\sum_{k=1}^{N}\left|h_{t d}\left(t_{k}\right)\right|^{2} e^{i \delta \omega t_{k}}\right\rangle\right. \\
& \left.+\left\langle\sum_{k=1}^{N} \sum_{j=1, j \neq k}^{N} h_{t d}\left(t_{k}\right) h_{t d}^{*}\left(t_{j}\right) e^{i(\omega+\delta \omega / 2) t_{k}} e^{-i(\omega-\delta \omega / 2) t_{j}}\right\rangle\right) .
\end{aligned}
$$

By using the hypothesis that the arrival times are independent, and that they are described by the density probability function $f(t)$, Eq. (A7) yields

$$
\begin{aligned}
\left\langle\tilde{E}(\omega+\delta \omega / 2) \tilde{E}^{*}(\omega-\delta \omega / 2)\right\rangle= & e^{2} \tilde{H}_{t i}(\omega+\delta \omega / 2) \tilde{H}_{t i}^{*}(\omega-\delta \omega / 2) N \\
& \times \int_{-\infty}^{+\infty}\left|h_{t d}\left(t_{k}\right)\right|^{2} e^{i \delta \omega t_{k}} f\left(t_{k}\right) d t_{k}+e^{2} \tilde{H}_{t i}(\omega+\delta \omega / 2) \tilde{H}_{t i}^{*}(\omega-\delta \omega / 2) N(N-1) \\
& \times \int_{-\infty}^{+\infty} h_{t d}\left(t_{k}\right) f\left(t_{k}\right) e^{i(\omega+\delta \omega / 2) t_{k}} d t_{k} \int_{-\infty}^{+\infty} h_{t d}^{*}\left(t_{j}\right) f\left(t_{j}\right) e^{-i(\omega-\delta \omega / 2) t_{j}} d t_{j} .
\end{aligned}
$$

The second term on right-hand side is negligible with respect to the first one. It can be shown by using the same arguments presented in [15], while discussing the spectral correlation of the first order:

$$
\left\langle\tilde{E}(\omega+\delta \omega / 2) \tilde{E}^{*}(\omega-\delta \omega / 2)\right\rangle \approx e^{2} N \tilde{H}_{t i}(\omega+\delta \omega / 2) \tilde{H}_{t i}^{*}(\omega-\delta \omega / 2) \int_{-\infty}^{+\infty}\left|h_{t d}(t)\right|^{2} f(t) e^{i \delta \omega t} d t
$$

We now calculate the spectral correlation of the second order: 


$$
\begin{aligned}
\left\langle|E(\omega+\delta \omega / 2)|^{2}|E(\omega-\delta \omega / 2)|^{2}\right\rangle= & e^{4}\left|\tilde{H}_{t i}(\omega+\delta \omega / 2)\right|^{2}\left|\tilde{H}_{t i}(\omega-\delta \omega / 2)\right|^{2}\left\langle\sum_{k=1}^{N} \sum_{j=1}^{N} \sum_{l=1}^{N} \sum_{m=1}^{N} h_{t d}\left(t_{k}\right) h_{t d}^{*}\left(t_{j}\right) h_{t d}\left(t_{l}\right) h_{t d}^{*}\left(t_{m}\right)\right. \\
& \left.\times e^{i(\omega+\delta \omega / 2) t_{k}} e^{-i(\omega+\delta \omega / 2) t_{j}} e^{i(\omega-\delta \omega / 2) t_{l}} e^{-i(\omega-\delta \omega / 2) t_{m}}\right\rangle .
\end{aligned}
$$

As clarified in [15], the most contributing terms are $(k=j) \wedge(l=m) \wedge(k \neq l),(k=m) \wedge(j=l) \wedge(k \neq j)$. Since the arrival times are independent, we have

$$
\frac{\left\langle|E(\omega+\delta \omega / 2)|^{2}|E(\omega-\delta \omega / 2)|^{2}\right\rangle}{e^{4}\left|\tilde{H}_{t i}(\omega+\delta \omega / 2)\right|^{2}\left|\tilde{H}_{t i}(\omega-\delta \omega / 2)\right|^{2}} \approx \sum_{k=1}^{N} \sum_{l=1, l \neq k}^{N}\left\langle\left|h_{t d}\left(t_{k}\right)\right|^{2}\right\rangle\left\langle\left|h_{t d}\left(t_{l}\right)\right|^{2}\right\rangle+\sum_{k=1}^{N} \sum_{j=1, j \neq k}^{N}\left\langle\left|h_{t d}\left(t_{k}\right)\right|^{2} e^{i \delta \omega t_{k}}\right\rangle\left\langle\left|h_{t d}\left(t_{j}\right)\right|^{2} e^{\left.-i \delta \omega t_{j}\right\rangle}\right.
$$

yielding

$$
\frac{\left\langle|E(\omega+\delta \omega / 2)|^{2}|E(\omega-\delta \omega / 2)|^{2}\right\rangle}{e^{4} N(N-1)\left|\tilde{H}_{t i}(\omega+\delta \omega / 2)\right|^{2}\left|\tilde{H}_{t i}(\omega-\delta \omega / 2)\right|^{2}}=\left(\int_{-\infty}^{+\infty}\left|h_{t d}(t)\right|^{2} f(t) d t\right)^{2}+\left.\left.\left|\int_{-\infty}^{+\infty}\right| h_{t d}(t)\right|^{2} f(t) e^{i \delta \omega t} d t\right|^{2} .
$$

Equation (A12) together with (A9) proves Eq. (10).

We now consider the average profile $X(t)=\left\langle|E(t)|^{2}\right\rangle$ and its Fourier transform

$$
\tilde{X}(\delta \omega)=\int_{-\infty}^{\infty}\left\langle\left|E(t)^{2}\right|\right\rangle e^{-i \delta \omega t}=\frac{1}{2 \pi} \int_{-\infty}^{+\infty}\left\langle\tilde{E}\left(\omega_{a}-\delta \omega / 2\right) \tilde{E}^{*}\left(\omega_{a}+\delta \omega / 2\right)\right\rangle d \omega_{a} .
$$

The squared modulus of $\tilde{X}$ can be written as

$$
|\tilde{X}(\delta \omega)|^{2}=\frac{1}{4 \pi^{2}} \int_{-\infty}^{+\infty} \int_{-\infty}^{+\infty}\left\langle\tilde{E}\left(\omega_{a}-\delta \omega / 2\right) \tilde{E}^{*}\left(\omega_{a}+\delta \omega / 2\right)\right\rangle\left\langle\tilde{E}^{*}\left(\omega_{b}-\delta \omega / 2\right) \tilde{E}\left(\omega_{b}+\delta \omega / 2\right)\right\rangle d \omega_{a} d \omega_{b}
$$

By doing the substitution $\left\{\omega_{a}, \omega_{b}\right\} \rightarrow\{\omega, b\}$, so that $\omega_{a}=\omega+b / 2$ and $\omega_{b}=\omega-b / 2$, one obtains

$$
\begin{aligned}
|\tilde{X}(\delta \omega)|^{2}= & \frac{1}{4 \pi^{2}} \int_{-\infty}^{+\infty} d \omega \int_{-\infty}^{+\infty}\left\langle\tilde{E}(\omega+b / 2-\delta \omega / 2) \tilde{E}^{*}(\omega+b / 2+\delta \omega / 2)\right\rangle \\
& \times\left\langle\tilde{E}^{*}(\omega-b / 2-\delta \omega / 2) \tilde{E}(\omega-b / 2+\delta \omega / 2)\right\rangle d b .
\end{aligned}
$$

From Eq. (A15) by using Eqs. (A6) and (A9), neglecting $e^{4} N^{2}$ terms and with $\sigma_{a}=\frac{1}{\sqrt{3} \sigma_{h t}}$, we have

$$
\begin{aligned}
4 \pi^{2}|\tilde{X}(\delta \omega)|^{2} & =\left.\left.\int_{-\infty}^{+\infty} \frac{2 \sqrt{\pi} e^{-\left[\delta \omega^{2}+3\left(\omega-\omega_{0}\right)^{2} / 3 \sigma_{a}^{2}\right]}}{3 \sigma_{a}^{2}} d \omega\left|\int_{-\infty}^{+\infty}\right| h_{t d}(t)\right|^{2} f(t) e^{i \delta \omega t} d t\right|^{2} \\
& =\left.\left.\frac{2 \pi e^{-\left(\delta \omega^{2} / 3 \sigma_{a}^{2}\right)}}{3 \sigma_{a}^{2}}\left|\int_{-\infty}^{+\infty}\right| h_{t d}(t)\right|^{2} f(t) e^{i \delta \omega t} d t\right|^{2} .
\end{aligned}
$$

Equation (A2) is evaluated as

$$
W(\omega)=\frac{e^{-\left[\left(\omega-\omega_{0}\right)^{2} / \sigma_{a}^{2}+\sigma_{m}^{2}\right]}}{\sqrt{\pi} \sqrt{\sigma_{a}^{2}+\sigma_{m}^{2}}},
$$

and from Eq. (A5) by using Eq. (A12)

$$
G_{2}(\delta \omega)=\int_{-\infty}^{+\infty} W(\omega) d \omega \int_{-\infty}^{+\infty} e^{-\left\{\left[\Delta \sigma_{a}^{2}+(\Delta+\delta \omega) \sigma_{m}^{2}\right]^{2} / 4 \sigma_{a}^{2} \sigma_{m}^{2}\left(\sigma_{a}^{2}+\sigma_{m}^{2}\right)\right\}} \frac{\left.\left.\sqrt{\sigma_{a}^{2}+\sigma_{m}^{2}}\left|\int_{-\infty}^{+\infty}\right| h_{t d}(t)\right|^{2} f(t) e^{i(\Delta+\delta \omega) t} d t\right|^{2}}{\left.\left.2 \sqrt{\pi} \sigma_{a} \sigma_{m}\left|\int_{-\infty}^{+\infty}\right| h_{t d}(t)\right|^{2} f(t)\right|^{2}} d \Delta .
$$

We can substitute Eqs. (A16) and (A17) into (A18) and integrate over $\omega$ obtaining

$$
G_{2}(\delta \omega)=\int_{-\infty}^{+\infty} e^{-\left\{\left[\Delta \sigma_{a}^{2}+(\Delta+\delta \omega) \sigma_{m}^{2}\right]^{2} / 4 \sigma_{a}^{2} \sigma_{m}^{2}\left(\sigma_{a}^{2}+\sigma_{m}^{2}\right)\right\}} \frac{\sqrt{\sigma_{a}^{2}+\sigma_{m}^{2}} e^{\left[(\Delta+\delta \omega)^{2} / 3 \sigma_{a}^{2}\right]}|\tilde{X}(\Delta+\delta \omega)|^{2}}{2 \sqrt{\pi} \sigma_{a} \sigma_{m}|\tilde{X}(0)|^{2}} d \Delta .
$$

By choosing a particular shape of the average profile, one can find an analytical expression for the $G_{2}$ function. In the case of a Gaussian profile, with rms length $\sigma_{t}$, Eq. (A19) under the condition $\sigma_{t}>\frac{1}{2 \sqrt{3} \sigma_{a}}$ gives 


$$
\frac{e^{-\left[\delta \omega^{2} \sigma_{a}^{2}\left(3 \sigma_{a}^{2} \sigma_{t}^{2}-1\right)\right] /\left\{\left(\sigma_{a}^{2}+\sigma_{m}^{2}\right)\left[3 \sigma_{a}^{2}\left(1+4 \sigma_{m}^{2} \sigma_{t}^{2}\right)-\sigma_{m}^{2}\right]\right\}}}{\sqrt{\frac{3 \sigma_{a}^{2}\left(1+4 \sigma_{m}^{2} \sigma_{t}^{2}\right)-\sigma_{m}^{2}}{3\left(\sigma_{a}^{2}+\sigma_{m}^{2}\right)}}} .
$$

Equation (A20) can be simplified when $\sigma_{t} \gg \frac{1}{\sqrt{3} \sigma_{a}}$, where $\sigma_{t}$ is the rms duration of the average profile, yielding

$$
G_{2}(\delta \omega)=\int_{-\infty}^{+\infty} \frac{e^{-\left(\xi-\delta \omega \xi_{0}\right)^{2} / 2 \sigma^{2}}|\tilde{X}(\xi)|^{2}}{\sqrt{2 \pi} \sigma|\tilde{X}(0)|^{2}} d \xi,
$$

where $\sigma=\sqrt{2}\left(\sigma_{a} \sigma_{m} / \sqrt{\sigma_{a}^{2}+\sigma_{m}^{2}}\right)$ and $\xi_{0}=\sigma_{a}^{2} /\left(\sigma_{a}^{2}+\sigma_{m}^{2}\right)$.

\section{APPENDIX B: COMPARISON BETWEEN GAUSSIAN AND FLATTOP MODEL}

We can rewrite explicitly Eqs. (14) and (15) by using the variables

$$
\Omega=\frac{\delta \omega}{\sigma_{m}}, \quad T_{g}=\sigma_{t} \sigma_{m}, \quad T_{f}=T \sigma_{m},
$$

and we obtain

$$
\begin{gathered}
G_{2}^{g}(\Omega)=\frac{e^{-\left(\Omega^{2} T_{g}^{2} / \sqrt{1+4 T_{g}^{2}}\right)}}{\sqrt{1+4 T_{g}^{2}}}, \\
G_{2}^{f t}(\Omega)=\frac{e^{-\Omega^{2} / 4} \sqrt{\pi}}{2 T_{f}}\left[\operatorname{erf}\left(T_{f}-\frac{i \Omega}{2}\right)+\text { c.c. }\right]+\frac{e^{-T_{f}^{2} \cos T_{f} \Omega}}{T_{f}^{2}}-\frac{e^{-\Omega^{2} / 4} \Omega \sqrt{\pi}}{4 T_{f}^{2}}\left[i \operatorname{erf}\left(T_{f}-\frac{i \Omega}{2}\right)-\text { c.c. }\right] \\
-\frac{2-i \Omega \sqrt{\pi} e^{-\left(\Omega^{2} / 4\right)} \operatorname{erf}\left(i \frac{\Omega}{2}\right)}{2 T_{f}^{2}} .
\end{gathered}
$$

Now, we look for the relation between $T_{f}$ and $T_{g}$ giving the same number of modes $\frac{1}{G_{2}(0)}$ within the two different models. Such value of $T_{g}$ is found as a function of $T_{f}$ :

$$
T_{g}=\frac{1}{2} \sqrt{\frac{e^{2 T_{f}^{2}} T_{f}^{4}-1}{\left(1+e^{T_{f}^{2}}\left[\sqrt{\pi} T_{f} \operatorname{erf}\left(T_{f}\right)-1\right)\right]^{2}}} .
$$

The behavior of $\frac{T_{f}}{T_{g}\left(T_{f}\right)}$ is represented in Fig. 10, together with the values at the extremes of the domain

$$
\lim _{T_{f} \rightarrow 0} \frac{T_{f}}{T_{g}\left(T_{f}\right)}=\sqrt{12} \quad \lim _{T_{f} \rightarrow \infty} \frac{T_{f}}{T_{g}\left(T_{f}\right)}=2 \sqrt{\pi} .
$$

It turns out that $T_{f}=\sqrt{12} T_{g}$ is a very good approximation for the condition to have the same number of modes in pulses described by the above profiles for any value of $T_{f}$. When the number of modes is large, the asymptotic expressions for the $G_{2}$ functions can be written as

$$
\begin{gathered}
G_{2}^{g}(\Omega)=\frac{e^{-\left(\Omega^{2} / 4\right)}}{2 T_{g}}+\frac{\left(\Omega^{2}-2\right) e^{-\left(\Omega^{2} / 4\right)}}{32 T_{g}^{3}}+o\left(\frac{1}{T_{g}^{4}}\right), \\
G_{2}^{f t}(\Omega)=\frac{\sqrt{\pi} e^{-\left(\Omega^{2} / 4\right)}}{T_{f}}+\frac{-2+\sqrt{\pi} \operatorname{erfi}\left(\frac{\Omega}{2}\right) \Omega e^{-\left(\Omega^{2} / 4\right)}}{2 T_{f}^{2}}+o\left(\frac{1}{T_{g}^{4}}\right) .
\end{gathered}
$$

It shows that, for both models, the main contributing terms have Gaussian shapes with the same rms, which depend only on the spectrometer resolution $\sigma_{m}$. 


\section{APPENDIX C: $G_{2}$ DERIVATION WITHOUT USING THE LINEAR AMPLIFIER MODEL}

As it was shown by our simulations, and in [15] even in the nonlinear regime, Eq. (10) is nearly valid. We write it in the form

$$
\left\langle\left|\tilde{E}\left(\omega_{a}\right)\right|^{2}\left|\tilde{E}\left(\omega_{b}\right)\right|^{2}\right\rangle \approx\left\langle\tilde{E}\left(\omega_{a}\right) \tilde{E}^{*}\left(\omega_{b}\right)\right\rangle\left\langle\tilde{E}^{*}\left(\omega_{a}\right) \tilde{E}\left(\omega_{b}\right)\right\rangle+\left\langle\left|\tilde{E}\left(\omega_{a}\right)\right|^{2}\right\rangle\left\langle\left|\tilde{E}\left(\omega_{b}\right)\right|^{2}\right\rangle .
$$

This allows us to write Eq. (A5) as

$$
\begin{aligned}
G_{2}(\delta \omega)= & \int_{-\infty}^{+\infty} W(\omega) \\
& \times \frac{\int_{-\infty}^{+\infty} \int_{-\infty}^{+\infty} e^{\left(-\Delta^{2} / 4 \sigma_{m}^{2}\right)} e^{\left(-\Omega^{2} / \sigma_{m}^{2}\right)}\left|\left\langle\tilde{E}(\omega+\Omega+\delta \omega / 2+\Delta / 2) \tilde{E}^{*}(\omega+\Omega-\delta \omega / 2-\Delta / 2)\right\rangle\right|^{2} d \Delta d \Omega}{\int_{-\infty}^{+\infty} \int_{-\infty}^{+\infty} e^{\left(-\Delta^{2} / 4 \sigma_{m}^{2}\right)} e^{\left(-\Omega^{2} / \sigma_{m}^{2}\right)}\left\langle|\tilde{E}(\omega+\Omega+\delta \omega / 2+\Delta / 2)|^{2}\right\rangle\left\langle\left.\tilde{E}(\omega+\Omega-\delta \omega / 2-\Delta / 2)\right|^{2}\right\rangle d \Delta d \Omega} d \omega
\end{aligned}
$$

If the spectrometer resolution function is much narrower than the FEL bandwidth, then we use the following approximation:

$$
\int_{-\infty}^{+\infty} e^{-\left(x^{2} / 2 \sigma_{m}^{2}\right)}\left\langle|E(\omega+x)|^{2}\right\rangle d x \approx \int_{-\infty}^{+\infty} e^{-\left(x^{2} / 2 \sigma_{m}^{2}\right)}\left\langle|E(\omega)|^{2}\right\rangle d \Omega
$$

By using this Eq. (C3) and Eq. (A15), we can rewrite expressions (C2) and (A2) as

$$
\begin{gathered}
G_{2}(\delta \omega)=\int_{-\infty}^{+\infty} W(\omega) \frac{\int_{-\infty}^{+\infty} \int_{-\infty}^{+\infty} e^{-\Delta^{2} / 4 \sigma_{m}^{2}} e^{-\Omega^{2} / \sigma_{m}^{2}}\left|\left\langle\tilde{E}(\omega+\Omega+\delta \omega / 2+\Delta / 2) \tilde{E}^{*}(\omega+\Omega-\delta \omega / 2-\Delta / 2)\right\rangle\right|^{2} d \Delta d \Omega}{2 \pi \sigma_{m}^{2}\left\langle|\tilde{E}(\omega+\delta \omega / 2)|^{2}\right\rangle\left\langle\left.\tilde{E}(\omega-\delta \omega / 2)\right|^{2}\right\rangle} d \omega \\
W(\omega)=\frac{\int_{-\infty}^{+\infty}\left\langle|E(\omega-b / 2)|^{2}\right\rangle\left\langle|E(\omega+b / 2)|^{2}\right\rangle}{4 \pi^{2}|\tilde{X}(0)|^{2}}
\end{gathered}
$$

We introduce $g_{1}$ formalism as in Eq. (2) and rewrite Eqs. (C4) and (A15) as

$$
\begin{aligned}
& G_{2}(\delta \omega)=\int_{-\infty}^{\infty} \int_{-\infty}^{\infty} \int_{-\infty}^{+\infty} \int_{-\infty}^{+\infty} d \omega d b d \Delta d \Omega e^{-\Delta^{2} / 4 \sigma_{m}^{2}} e^{-\Omega^{2} / \sigma_{m}^{2}}\left|g_{1}(\omega+\Omega, \delta \omega+\Delta)\right|^{2} \\
& \times \frac{\left\langle\left|\tilde{E}^{*}(\omega+\Omega-\Delta / 2-\delta \omega / 2)\right|^{2}\right\rangle\left\langle\left|\tilde{E}^{*}(\omega+\Omega+\Delta / 2+\delta \omega / 2)\right|^{2}\right\rangle}{2 \pi \sigma_{m}^{2}\left\langle|\tilde{E}(\omega+\delta \omega / 2)|^{2}\right\rangle\left\langle|\tilde{E}(\omega-\delta \omega / 2)|^{2}\right\rangle} \frac{\left\langle\left. E(\omega-b / 2)\right|^{2}\right\rangle\left\langle|E(\omega+b / 2)|^{2}\right\rangle}{4 \pi^{2}|\tilde{X}(0)|^{2}} \\
&|\tilde{X}(\delta \omega)|^{2}= \frac{1}{4 \pi^{2}} \int_{-\infty}^{+\infty} d \omega \int_{-\infty}^{+\infty} g_{1}(\omega+b / 2, \delta \omega) g_{1}^{*}(\omega-b / 2, \delta \omega) \\
& \times \sqrt{\left\langle|\tilde{E}(\omega-b / 2-\delta \omega / 2)|^{2}\right\rangle\left\langle|\tilde{E}(\omega-b / 2+\delta \omega / 2)|^{2}\right\rangle} \\
& \times \sqrt{\left\langle|\tilde{E}(\omega+b / 2-\delta \omega / 2)|^{2}\right\rangle\left\langle\left.\tilde{E}(\omega+b / 2+\delta \omega / 2)\right|^{2}\right\rangle} d b .
\end{aligned}
$$

Equation (C6) can be simplified by using the approximation (C3) on $\Delta$ and $\Omega$ :

$$
G_{2}(\delta \omega)=\int_{-\infty}^{\infty} \int_{-\infty}^{\infty} \int_{-\infty}^{\infty} \int_{-\infty}^{\infty} \frac{e^{-\Delta^{2} / 4 \sigma_{m}^{2}} e^{-\Omega^{2} / \sigma_{m}^{2}}\left|g_{1}(\omega+\Omega, \delta \omega+\Delta)\right|^{2}\left\langle|E(\omega-b / 2)|^{2}\right\rangle\left\langle|E(\omega+b / 2)|^{2}\right\rangle}{2 \pi \sigma_{m}^{2} 4 \pi^{2}|\tilde{X}(0)|^{2}} d \omega d b d \Delta d \Omega
$$

and in a similar way, Eq. (C7) can be simplified when the average spectral spike width is much narrower than the FEL bandwidth as

$$
|\tilde{X}(\delta \omega)|^{2}=\frac{1}{4 \pi^{2}} \int_{-\infty}^{+\infty} d \omega \int_{-\infty}^{+\infty} g_{1}(\omega+b / 2, \delta \omega) g_{1}^{*}(\omega-b / 2, \delta \omega)\left\langle|\tilde{E}(\omega-b / 2)|^{2}\right\rangle\left\langle|\tilde{E}(\omega+b / 2)|^{2}\right\rangle d b .
$$

Further we approximate 


$$
\begin{gathered}
\int_{-\infty}^{+\infty} d \omega \int_{-\infty}^{+\infty} g_{1}(\omega+b / 2, \delta \omega) g_{1}^{*}(\omega-b / 2, \delta \omega)\left\langle|\tilde{E}(\omega-b / 2)|^{2}\right\rangle\left\langle|\tilde{E}(\omega+b / 2)|^{2}\right\rangle d b \\
\approx \int_{-\infty}^{+\infty} d \omega \int_{-\infty}^{+\infty}\left|g_{1}(\omega, \delta \omega)\right|^{2}\left\langle|\tilde{E}(\omega-b / 2)|^{2}\right\rangle\left\langle|\tilde{E}(\omega+b / 2)|^{2}\right\rangle d b .
\end{gathered}
$$

The expression is exact for $\delta \omega=0$ but it is approximated, in general, for other values of $\delta \omega$. Referring to the exponential growth model, from Eq. (A9) we have

$$
\begin{aligned}
& g_{1}(\omega+b / 2, \delta \omega) g_{1}^{*}(\omega-b / 2, \delta \omega) \\
& =e^{-i\left(1 / 2 \sigma_{a}^{2}\right)(b / \sqrt{3}) \delta \omega} \frac{\left.\left.\left|\int_{-\infty}^{+\infty}\right| h_{t d}(t)\right|^{2} f(t) e^{i \delta \omega t} d t\right|^{2}}{\left.\left.\left|\int_{-\infty}^{+\infty}\right| h_{t d}(t)\right|^{2} f(t) d t\right|^{2}} .
\end{aligned}
$$

Equation $(\mathrm{C} 11)$ is not a function of $\omega$, and its width in the variable $\delta \omega$ is of the order of $1 / T$, where $T$ is the characteristic length of the profile. From Eq. (C10), if $b$ is larger than the FEL bandwidth, then $\left\langle|\tilde{E}(\omega-b / 2)|^{2}\right\rangle \times$ $\left\langle|\tilde{E}(\omega+b / 2)|^{2}\right\rangle$ becomes close to zero. Inside this bounded region for $b$ and $\delta \omega$ the phase of exponential factor in Eq. (C11) is approximately zero when $1 / T \ll \sigma_{a}$, showing that, within these assumptions, Eq. (C10) is valid. An important result found in [15] is that the spectral correlation functions do not change considerably between the exponential growth and the saturation regime. This means that $g_{1}(\omega, \delta \omega)$ has a width in $\delta \omega$ of the order of $1 / T$ also in the saturation regime. First, the approximation (C10) requires an assumption that the phase of $g_{1}(\omega-$ $b / 2, \delta \omega) g_{1}^{*}(\omega-b / 2, \delta \omega)$ is nearly zero for $\delta \omega$ being inside the described region, and for $b$ inside the FEL bandwidth. Second, it requires that the amplitude of $g_{1}(\omega, \delta \omega)$ changes slowly in the $\omega$ variable. By using the approximation $(\mathrm{C} 10)$ we obtain

$$
\begin{gathered}
G_{2}(\delta \omega)=\int_{-\infty}^{\infty} \int_{-\infty}^{\infty} \int_{-\infty}^{\infty} \frac{e^{-\Delta^{2} / 4 \sigma_{m}^{2}}\left|g_{1}(\omega, \delta \omega+\Delta)\right|^{2}\left\langle|E(\omega-b / 2)|^{2}\right\rangle\left\langle|E(\omega+b / 2)|^{2}\right\rangle}{2 \sqrt{\pi} \sigma_{m} 4 \pi^{2}|\tilde{X}(0)|^{2}} d \omega d b d \Delta \\
|\tilde{X}(\delta \omega)|^{2}=\frac{1}{4 \pi^{2}} \int_{-\infty}^{+\infty} d \omega \int_{-\infty}^{+\infty}\left|g_{1}(\omega, \delta \omega)\right|^{2}\left\langle|\tilde{E}(\omega-b / 2)|^{2}\right\rangle\left\langle|\tilde{E}(\omega+b / 2)|^{2}\right\rangle d b .
\end{gathered}
$$

By substituting (C13) into (C12) we finally obtain

$$
G_{2}(\delta \omega)=\int_{-\infty}^{\infty} \frac{e^{-\Delta^{2} / 4 \sigma_{m}^{2}}|\tilde{X}(\delta \omega+\Delta)|^{2}}{2 \sqrt{\pi} \sigma_{m}|\tilde{X}(0)|^{2}} d \Delta .
$$

For $\sigma_{a} \gg \sigma_{m}$ Eq. (A21) gives, as expected, Eq. (C14).

[1] P. Emma et al., Nature Photon. 4, 641 (2010).

[2] Z. Huang and K.-J. Kim, Phys. Rev. ST Accel. Beams 10, 034801 (2007).

[3] M. Zolotorev and G. Stupakov, in Proceedings of the Particle Accelerator Conference, Vancouver, BC, Canada, 1997 (IEEE, New York, 1997), p. 2180.

[4] J. Krzywinsky, E. L. Saldin, E. A. Schneidmiller, and M. V. Yurkov, Nucl. Instrum. Methods Phys. Res., Sect. A 401, 429 (1997).

[5] P. Catravas et al., Phys. Rev. Lett. 82, 5261 (1999).

[6] V. Sajaev, in Proceedings of the European Particle Accelerator Conference, Vienna, 2000 (EPS, Geneva, 2000), p. 1806.

[7] F. Sannibale, G. V. Stupakov, M.S. Zolotorev, D. Filippetto, and L. Jägerhofer, Phys. Rev. ST Accel. Beams 12, 032801 (2009).

[8] M. Yabashi, K. Tamasaku, and T. Ishikawa, Phys. Rev. Lett. 88, 244801 (2002).

[9] W. Ackermann et al., Nature Photon. 1, 336 (2007).

[10] J. Wu et al., in Proceedings of FEL2010, Malmö, 2010 [http://accelconf.web.cern.ch/AccelConf/FEL2010/html/ sessi0n.htm], MOPC14, p. 147.
[11] E. L. Saldin, E. A. Schneidmiller, and M. V. Yurkov, The Physics of Free Electron Lasers (Springer, Berlin, 2000).

[12] S. Krinsky, Phys. Rev. E 69, 066503 (2004).

[13] P. Emma, K. Bane, M. Cornacchia, Z. Huang, H. Schlarb, G. Stupakov, and D. Walz, Phys. Rev. Lett. 92, 07801 (2004)

[14] J. W. Goodman, Statistical Optics (John Wiley \& Sons, Inc., New York, 1985).

[15] E. L. Saldin, E. A. Schneidmiller, and M. V. Yurkov, Opt. Commun. 148, 383 (1998).

[16] P. Heimann et al., Rev. Sci. Instrum. 82, 093104 (2011).

[17] Y. T. Ding, Z. Huang, and S. A. Ocko, in Proceedings of FEL2010, Malmö, 2010 [http://accelconf.web.cern.ch/ AccelConf/FEL2010/html/sessi0n.htm], MOPC16, p. 151.

[18] S. Reiche, Nucl. Instrum. Methods Phys. Res., Sect. A 429, 243 (1999).

[19] I. Vartaniants et al., Phys. Rev. Lett. 107, 144801 (2011).

[20] P. Heimann (private communication).

[21] P. Emma, Z. Huang, and M. Borland, in Proceedings of FEL2004 (Comitato Conferenze Elettra, Trieste, Italy, 2004), p. 333. 